\title{
Noncommutative function theory and unique extensions
}

\author{
by \\ David P. Blecher (Houston, TX) and Louis E. Labuschagne (Unisa)
}

\begin{abstract}
We generalize, to the setting of Arveson's maximal subdiagonal subalgebras of finite von Neumann algebras, the Szegö $L^{p}$-distance estimate and classical theorems of F. and M. Riesz, Gleason and Whitney, and Kolmogorov. As a byproduct, this completes the noncommutative analog of the famous cycle of theorems characterizing the function algebraic generalizations of $H^{\infty}$ from the 1960's. A sample of our other results: we prove a Kaplansky density result for a large class of these algebras, and give a necessary condition for every completely contractive homomorphism on a unital subalgebra of a $C^{*}$-algebra to have a unique completely positive extension.
\end{abstract}

1. Introduction. Function algebras are subalgebras of $C(K)$ spaces, or equivalently, subalgebras of commutative $C^{*}$-algebras. Thus function algebras are examples of operator algebras (subalgebras of general $C^{*}$-algebras). With this in mind, much work has been done to transfer results or perspectives from function theory to operator algebraic settings. One such setting where this transfer is particularly striking, is the theory of noncommutative $H^{p}$ spaces associated with Arveson's maximal subdiagonal subalgebras of finite von Neumann algebras. Remarkably, many of the central results from abstract analytic function theory, and in particular much of the classical generalized $H^{p}$ function theory from the 1960's, may be generalized almost verbatim to subdiagonal algebras. The proofs in the noncommutative case however, while often modeled loosely on the "commutative" arguments of Helson and Lowdenslager [15] and others, usually require substantial input from the theory of von Neumann algebras and noncommutative $L^{p}$ spaces. This has been done for example in $[1,24,28,23,25,26,21,4,5,7]$. In fact

2000 Mathematics Subject Classification: Primary 46L51, 46L52, 47L75; Secondary 46J15, 46K50, 47L45.

Key words and phrases: subdiagonal operator algebra, noncommutative Hardy spaces, finite von Neumann algebras, unique extension, F \& M Riesz.

Blecher is partially supported by grant DMS 0400731 from the National Science Foundation. Labuschagne is partially supported by the National Research Foundation, and conducted some of this research with the aid of a grant under the Poland-SA cooperation agreement. 
in many cases - like Szegö's theorem-completely new proofs have had to be invented.

In the present paper we tackle what appears to us to be the main "classical" results which have resisted generalization to date, namely those referred to in the generalized function theory literature from the 1960's as, respectively, the F. and M. Riesz, Gleason and Whitney, Szegö $L^{p}$, and Kolmogorov, theorems. As a byproduct, this together with the main theorem in [4], and some results in our other papers [5, 7], completes the noncommutative analog of the famous cycle of theorems characterizing the function algebraic generalizations of $H^{\infty}$ from the 1960's (see Theorem 1.2 in our survey [6] for a full statement of the cycle of results). In fact we are now at last able to make the following statement: all of the generalized $H^{p}$ function theory, as summarized in [30] for example, extends further to the setting of subdiagonal algebras.

In Arveson's setting, and we will use this notation in the rest of this paper, we have a weak* closed unital subalgebra $A$ of a von Neumann algebra $M$ with a faithful normal tracial state $\tau$ such that if $\Phi$ is the unique conditional expectation from $M$ onto $\mathcal{D}=A \cap A^{*}$ satisfying $\tau=\tau \circ \Phi$, then $\Phi$ is a homomorphism on $A$. Take note that here $A^{*}$ denotes the set $\left\{a: a^{*} \in A\right\}$ and not the Banach dual of $A$. For the sake of clarity we will write $X^{\star}$ for the Banach dual of a normed space $X$. We say that a subalgebra $A$ of the type described above is a tracial subalgebra of $M$. If in addition $A+A^{*}$ is weak ${ }^{*}$ dense in $M$ then we say that $A$ is maximal subdiagonal (see $[1,10]$ ). A large number of very interesting examples of these objects were given by Arveson [1] and others (see e.g. [33, 24]). If $\mathcal{D}$ is one-dimensional we say that $A$ is antisymmetric; if further $M$ is commutative then $A$ is called a weak* Dirichlet algebra [30]. Surprisingly, for antisymmetric maximal subdiagonal algebras, many of the "commutative" proofs from [30] require almost no change at all! It is worth saying that classical notions of "analyticity" correspond in some very vague sense to the case that $\mathcal{D}$ is "small". Indeed, if $A=M$ then $\mathcal{D}=M$ and $\Phi$ is the identity map, so that the theory essentially collapses to the theory of finite von Neumann algebras, which is clearly far away from classical concepts of "analyticity". Thus the reader should not be surprised that some of our theorems require as a hypothesis that $\mathcal{D}$ be small. Indeed, for our $\mathrm{F}$. and M. Riesz theorem to hold, we show that it is necessary and sufficient for $\mathcal{D}$ to be finite-dimensional. Because of this, in several applications of this theorem we assume $\operatorname{dim}(\mathcal{D})<\infty$.

A subsidiary theme in our paper is "unique extensions" of maps on $A$. We begin with some results on this topic in Section 2. Recall from [4] that a subalgebra $A$ of $M$ has the unique normal state extension property if there is a unique normal state on $M$ extending $\tau_{\mid A}$. If, on the other hand, for 
every state $\omega$ of $M$ with $\omega \circ \Phi=\omega$ on $A$, we always have $\omega \circ \Phi=\omega$ on $M$, then we say that $A$ has the $\Phi$-state property $\left({ }^{1}\right)$. The major unresolved question in [4] was whether a tracial subalgebra with the unique normal state extension property is maximal subdiagonal. We make what we feel is substantial progress on this question. In particular, we show that the question is equivalent to the question of whether every tracial subalgebra with the $\Phi$-state property is maximal subdiagonal, and equivalent to whether every tracial subalgebra satisfying a certain variant of the well known "factorization" property actually has "factorization". In Section 2 we also give an interesting necessary condition for completely contractive homomorphisms to have a unique completely positive extension. Our unique extension results play a role in the proof of our F. and M. Riesz theorem in Section 3, and are the primary thrust of the Gleason-Whitney theorem in Section 4. In Section 5 we prove our Szegö $L^{p}$ formula, and generalized Kolmogorov theorem.

Historically, the first noncommutative F. and M. Riesz theorem for subdiagonal algebras was the pretty theorem of Exel in [11]. This result assumes norm density $\left({ }^{2}\right)$ of $A+A^{*}$, and antisymmetry. (We are aware of the $\mathrm{F}$. and M. Riesz theorem of Arveson [2] and Zsido's extension thereof [33], but this result is quite distinct from the ones discussed above.) Although some of the steps of our proof parallel those of [11], the arguments are for the most part quite different. Indeed, generally in our paper the proofs will be modeled on the classical ones, but do however require some rather delicate additional machinery.

Finally, we remark that there are other, more recent, noncommutative variants of $H^{\infty}$ besides the subdiagonal algebras - see e.g. [27] and references therein. Although here too one finds noncommutative generalizations of classical $H^{p}$-theoretic results, such as the Szegö infimum theorem, these variants are in general quite unrelated, with only a formal correspondence to the present context. Although this other theory is extremely important in its own right, it seems to be the theory of finite subdiagonal algebras that is more conditioned to the application of noncommutative $L^{p}$ space techniques. Having said this, we are not aware of analogues of any of the results from our present paper in that literature. In fact, as was pointed out to us by G. Popescu, there is as yet no clear noncommutative analogue of $L^{\infty}(\mathbb{T})$ in that approach, and also no clear analogue of $H^{p}$ for $p \notin\{2, \infty\}$. Thus it is not at all obvious how one could even formulate a Gleason-Whitney or F. and M. Riesz theorem in that context.

$\left({ }^{1}\right)$ One could replace states here by positive unital $B(H)$-valued maps, for a Hilbert space $H$, but this formulation is easily seen to be equivalent.

$\left({ }^{2}\right)$ This is perhaps an appropriate hypothesis for an F. and M. Riesz theorem, but unfortunately it does not cover the case of maximal subdiagonal algebras. 
2. Some results on unique extensions. For a functional $\omega \in M^{\star}$, we will need to compare the property $\omega=\omega \circ \Phi$ on $A$ with $\omega=\omega \circ \Phi$ on $M$. We begin with the following remarks. It is easy to see, since $\Phi$ is idempotent, that $\omega=\omega \circ \Phi$ on $A$ iff $A_{0} \subset \operatorname{Ker}(\omega)$. Here and throughout our paper, $A_{0}=A \cap \operatorname{Ker}(\Phi)$, a closed two-sided ideal in $A$.

For normal functionals one can say more, although this will not play an important role for us. If $f \in L^{1}(M)$ let $\omega_{f}=\tau(f \cdot)$. From the last paragraph, $\omega_{f}=\omega_{f} \circ \Phi$ on $A$ iff $\tau\left(f A_{0}\right)=(0)$. On the other hand, $\omega_{f}=\omega_{f} \circ \Phi$ on $M$ iff $\tau(f a)=\tau(f \Phi(a))=\tau(\Phi(f) a)$ for all $a \in M$ iff $f=\Phi(f)$ iff $f \in L^{1}(\mathcal{D})$. (For some of these relations the reader may need to look e.g. at the second paragraph of the proof of [4, Lemma 4.1].)

Proposition 2.1. If $A$ is a tracial subalgebra of $M$ then the unique normal state extension property is equivalent to the following property: whenever $\omega$ is a normal state of $M$ satisfying $\omega=\omega \circ \Phi$ on $A$, then $\omega=\omega \circ \Phi$ on $M$.

Proof. Suppose that $A$ has the unique normal state extension property, and that $\omega$ is a normal state of $M$ satisfying $\omega=\omega \circ \Phi$ on $A$. If $\omega=\tau(f \cdot)$, where $f \in L^{1}(M)_{+}$, then by the remarks preceding Proposition 2.1 we have $\tau\left(f A_{0}\right)=(0)$. Hence $f \in L^{1}(\mathcal{D})$ by $[4$, Lemma 4.1]. Hence $\omega=\omega \circ \Phi$ on $M$.

For the converse, note that if $g \in L^{1}(M)_{+}$with $\tau=\tau(g \cdot)$ on $A$, then since $\tau=\tau \circ \Phi$, we have $\tau(g \cdot)=\tau(g \cdot) \circ \Phi$ on $A$, and hence $\tau(g \cdot)=\tau(g \cdot) \circ \Phi$ on $M$. By the remarks above, $g \in L^{1}(\mathcal{D})_{+}$. But then the fact that $\tau=\tau(g \cdot)$ on $\mathcal{D}$ is enough to force $g=\mathbb{1}$. So $A$ has the unique normal state extension property.

We say that a subalgebra $A$ of $M$ has factorization if given $b \in M^{+} \cap M^{-1}$ we can find $a \in A^{-1}$ with $b=a^{*} a$ (or equivalently $b=a a^{*}$ ). It is shown in [1] that any maximal subdiagonal algebra has factorization. Thus it is logmodular, namely any such $b$ is a uniform limit of terms of the form $a^{*} a$ with $a \in A^{-1}$. In fact, in the category of tracial algebras, factorization or logmodularity are equivalent to maximal subdiagonality [4]. By the next result such algebras have a formally much stronger property than that of the last proposition:

TheOREm 2.2. Let $A$ be a logmodular subalgebra of a $C^{*}$-algebra $M$, and let $\Psi$ be a positive contractive projection from $M$ onto a subalgebra of $A$ containing $\mathbb{1}_{M}$, which is a homomorphism on $A$. Then for any state $\left({ }^{3}\right)$ $\omega$ of $M$, we have $\omega=\omega \circ \Psi$ on $M$ whenever $\omega=\omega \circ \Psi$ on $A$.

Proof. If $a \in A^{-1}$ then by hypothesis we have

$$
\omega\left(\Psi(a) a^{-1}\right)=\omega\left(\Psi\left(\Psi(a) a^{-1}\right)\right)=\omega\left(\Psi(a) \Psi\left(a^{-1}\right)\right)=\omega(\mathbb{1})=1 .
$$

$\left({ }^{3}\right)$ As before it is not difficult to see that one could here replace states by positive unital $B(H)$-valued maps. 
By the Cauchy-Schwarz and Kadison-Schwarz inequalities we deduce

$$
\begin{aligned}
1 & \leq \omega\left(\Psi(a) \Psi(a)^{*}\right) \omega\left(\left(a^{-1}\right)^{*} a^{-1}\right) \leq \omega\left(\Psi\left(a a^{*}\right)\right) \omega\left(\left(a^{-1}\right)^{*} a^{-1}\right) \\
& =\omega\left(\Psi\left(a a^{*}\right)\right) \omega\left(\left(a a^{*}\right)^{-1}\right) .
\end{aligned}
$$

We can now follow the proof of [8, Theorem 4.3.11] or [3, Theorem 4.4]. Since $A$ is logmodular, for any $b \in M^{-1} \cap M_{+}$we have $1 \leq \omega(\Psi(b)) \omega\left(b^{-1}\right)$. This leads to the inequality $1 \leq \omega\left(\Psi\left(e^{t u}\right)\right) \omega\left(e^{-t u}\right)=f(t)$ for $u \in M_{\mathrm{sa}}$. Differentiating and noting that $f^{\prime}(0)=0$ yields $\omega(u)=\omega(\Psi(u))$ as required.

When applied to tracial algebras and their associated canonical conditional expectations, the preceding result still holds under a formally weaker hypothesis. Specifically we say that a tracial subalgebra $A$ of $M$ with canonical conditional expectation $\Phi$ has conditional factorization if given any $b \in M_{+} \cap M^{-1}$, we have $b=|a|$ for some element $a \in A \cap M^{-1}$ with $\Phi(a) \Phi\left(a^{-1}\right)=1$.

Corollary 2.3. A tracial subalgebra of $M$ with conditional factorization has the $\Phi$-state property.

Proof. The proof of the preceding theorem readily adapts, upon replacing $a$ with $a^{-1}$ and $b$ with $b^{-1}$.

We say that $A$ has the unique state extension property if there is a unique state on $M$ extending $\tau_{\mid A}$. This property is formally weaker than the $\Phi$-state property:

Proposition 2.4. Let $A$ be a weak $k^{*}$ closed unital subalgebra of $M$. If $A$ has the $\Phi$-state property then it has the unique state extension property. The converse is true if $A$ is antisymmetric.

Proof. Suppose that $\omega$ is a state of $M$ extending $\tau_{\mid A}$. Then $\omega \circ \Phi=\tau \circ \Phi=$ $\tau=\omega$ on $A$. By the $\Phi$-state property, on $M$ we have $\omega=\omega \circ \Phi=\tau \circ \Phi=\tau$. For the converse we need only note that if $A$ is antisymmetric, then $\omega \circ \Phi=\omega$ on $A$ forces $\tau=\omega$ on $A$.

Corollary 2.5. Suppose that $A$ is a tracial subalgebra of $M$ with the unique normal state extension property. Then $A_{\infty}=M \cap[A]_{2}$ is a tracial subalgebra with the $\Phi$-state property.

Proof. First note that by [4, Theorem 4.4], $A_{\infty}$ is a tracial subalgebra of $M$ with respect to the same $\Phi$ and $\tau$. By [4, Theorem 4.6], $A_{\infty}$ has conditional factorization. Corollary 2.3 now gives the conclusion.

COROLlaRY 2.6. The open question from [4] as to whether every tracial subalgebra with the unique normal state extension property is maximal subdiagonal, is equivalent to the question of whether every tracial subalgebra with the $\Phi$-state property is maximal subdiagonal. It is also equivalent to whether every tracial subalgebra with the unique state extension property is 
maximal subdiagonal. It is also equivalent to whether every tracial subalgebra with conditional factorization has factorization.

Proof. Suppose that every tracial subalgebra with the $\Phi$-state property is maximal subdiagonal, and that $A$ has the unique normal state extension property. By Corollary $2.5, A_{\infty}$ has the $\Phi$-state property. Hence it is maximal subdiagonal, and therefore satisfies $L^{2}$ density. Consequently, $A$ satisfies $L^{2}$ density, and so $A$ is maximal subdiagonal by [4].

Similarly, suppose that every tracial subalgebra with conditional factorization has factorization, and suppose that $A$ has the $\Phi$-state property. By the results above, $A$ has the unique normal state extension property, and so by $\left[4\right.$, Theorem 4.6], $A_{\infty}$ has conditional factorization. By hypothesis, $A_{\infty}$ has factorization. Thus it is maximal subdiagonal by [4], and thus, as in the last paragraph, $A$ is maximal subdiagonal.

The other directions are obvious from the above.

REMARK. Since the factorization property has been well studied, we would guess that those more familiar than ourselves with factorization for concrete examples such as CSL algebras may be able to easily resolve the final question in the last corollary.

In [22], Lumer considered the property of "uniqueness of representing measure", namely that every multiplicative functional on $A \subset C(K)$ has a unique extension to a state on $C(K)$. He showed how this condition could be used as another possible axiom from which all the generalized $H^{p}$ theory may be derived. The natural noncommutative generalization of Lumer's property is that every completely contractive representation of $A$ has a unique completely positive extension to $M$. It is known that maximal subdiagonal algebras have this property [3, 8]. Although we have not settled the converse yet, we can say that every unital subalgebra of $M$ which has this property must in some sense be a large subalgebra of $M$. In this regard the following result represents some sort of converse to many of the preceding results which established various unique extension properties as a consequence of maximal subdiagonality.

In the following result we use the $C^{*}$-envelope $C_{\mathrm{e}}^{*}(A)$ of an operator algebra $A$. See e.g. [8, Section 4.3] for the definition of this, and for its universal property.

Theorem 2.7. Suppose that $A$ is a subalgebra of a unital $C^{*}$-algebra $B$ such that $\mathbb{1}_{B} \in A$, and suppose that for every Hilbert space $H$, every completely contractive unital homomorphism $\pi: A \rightarrow B(H)$ has a unique completely contractive (or equivalently completely positive) extension $B \rightarrow$ $B(H)$. Then $B=C_{\mathrm{e}}^{*}(A)$, the $C^{*}$-envelope of $A$. 
Proof. CASE 1: $A$ is a $C^{*}$-subalgebra of $B$. In this case, since contractive homomorphisms on $C^{*}$-algebras are $*$-homomorphisms (see e.g. [8, Proposition 1.2.4]), we must prove that if every unital $*$-homomorphism $\pi: A \rightarrow B(H)$ has a unique completely contractive extension $B \rightarrow B(H)$, then $A=B$. To see this, let $\varrho: B \rightarrow B(H)$ be the universal representation of $B$. Then $\varrho$ is unital, and hence so is $\pi=\varrho_{\mid A}$. Let $U$ be a unitary in $\pi(A)^{\prime}$. Since $U^{*} \varrho(\cdot) U=\varrho$ on $A$, by hypothesis we have $U^{*} \varrho(\cdot) U=\varrho$ on $B$, and thus $U \in \varrho(B)^{\prime}$. Thus $\pi(A)^{\prime}=\varrho(B)^{\prime}$, and it follows that $\pi(A)^{\prime \prime}=\varrho(B)^{\prime \prime}$. If $\widetilde{\varrho}$ is the unique normal extension of $\varrho$ to $B^{\star \star}$, then $\widetilde{\varrho}$ is faithful on $B^{\star \star}$ and it has range $\varrho(B)^{\prime \prime}$. The restriction of $\widetilde{\varrho}$ to the copy $A^{\perp \perp}$ of $A^{\star \star}$ inside $B^{\star \star}$ has range $\pi(A)^{\prime \prime}=\overline{\pi(A)}^{w^{*}}$, and is therefore surjective. This forces the copy of $A^{\star \star}$ inside $B^{\star \star}$ to be all of $B^{\star \star}$. Thus $A=B \cap A^{\perp \perp}=B$.

CASE 2: general. Let $C=C^{*}(A)$, the $C^{*}$-algebra generated by $A$ in $B$. Since $A \subset C$, it follows from the hypothesis that every unital $*$-homomorphism $\pi: C \rightarrow B(H)$ has a unique completely contractive extension $B \rightarrow B(H)$. By Case $1, C=B$.

By virtue of this fact, we need only prove that $C^{*}(A)=C_{\mathrm{e}}^{*}(A)$ under the assumptions of the theorem. By the universal property of $C_{\mathrm{e}}^{*}(A)$, there is a *-epimorphism $\theta: B=C^{*}(A) \rightarrow C_{\mathrm{e}}^{*}(A)$ restricting to the "identity map" on $A$. If $B \subset B(H)$ then the canonical map from the copy of $A$ in $C_{\mathrm{e}}^{*}(A)$ to $A \subset B(H)$ has a completely positive extension $\Phi: C_{\mathrm{e}}^{*}(A) \rightarrow B(H)$. On $A$, the map $\Phi \circ \theta$ is the identity map, so that by hypothesis $\Phi \circ \theta=i_{B}$. Thus $\theta$ is one-to-one, and hence $C^{*}(A)$ is the $C^{*}$-envelope of $A$.

Corollary 2.8. Suppose that $A$ is a tracial subalgebra of $M$ such that for every Hilbert space $H$, every completely contractive unital homomorphism $\pi: A \rightarrow B(H)$ has a unique completely contractive (or equivalently completely positive) extension $B \rightarrow B(H)$. Then $A$ generates $M$ as a $C^{*}$ algebra. Indeed, $M$ is the $C^{*}$-envelope of $A$.

3. A noncommutative F. and M. Riesz theorem. The classical form of the F. and M. Riesz theorem (see e.g. [18]) is known to fail for weak* Dirichlet algebras; and hence it will also fail for subdiagonal algebras. However, there is an equivalent version of the theorem which is true for weak* Dirichlet algebras [17, 30], and we will focus on this variant here. Namely, we shall say that a tracial subalgebra $A$ of $M$ has the $F \& B$ Riesz property if for every bounded functional $\left({ }^{4}\right) \varrho$ on $M$ which annihilates $A_{0}$, the normal and singular parts $\varrho_{\mathrm{n}}$ and $\varrho_{\text {s }}$ annihilate $A_{0}$ and $A$ respectively. During our investigation we shall have occasion to make use of the polar

$\left({ }^{4}\right)$ One could replace $\varrho$ here by a $B(H)$-valued map, for a Hilbert space $H$, but this formulation is easily seen to be equivalent. 
decomposition of normal functionals on a von Neumann algebra. We take the opportunity to point out that for our purposes we shall assume such a polar decomposition to be of the form $\omega(a)=|\omega|(u a)$ for some partial isometry $u$, rather than $\omega(a)=|\omega|(a u)$ which seems to be more common among the proponents of noncommutative $L^{p}$ spaces.

The following result shows that to study the F \& M Riesz property, we may restrict our attention to algebras for which the diagonal $\mathcal{D}$ is finitedimensional:

Proposition 3.1. If a tracial subalgebra $A$ of $M$ has the $F$ \& $M$ Riesz property then the diagonal $\mathcal{D}$ is finite-dimensional.

Proof. Let $\psi \in \mathcal{D}^{\star}$. Then $\psi \circ \Phi \in M^{\star}$ annihilates $A_{0}$. By the $\mathrm{F} \& \mathrm{M}$ Riesz property, $\psi \circ \Phi$ agrees with $(\psi \circ \Phi)_{\mathrm{n}}$ on $A$, and so $\psi=\psi \circ \Phi_{\mid \mathcal{D}}$ is weak* continuous on $\mathcal{D}$. Thus $\mathcal{D}$ is reflexive, and therefore finite-dimensional.

Lemma 3.2. Let $A$ be a maximal subdiagonal subalgebra of $M$. Let $\omega$ be a state of $M$, and let $\left(\pi_{\omega}, \mathfrak{h}_{\omega}, \Omega_{\omega}\right)$ be the GNS representation of $\omega$. Further, let $\Omega_{0}$ be the orthogonal projection of $\Omega_{\omega}$ onto the closed subspace $\overline{\pi_{\omega}\left(A_{0}\right) \Omega_{\omega}}$.

(a) (i) There exists a central projection $p_{0}$ in $\pi_{\omega}(M)^{\prime \prime}$ such that for any $\xi, \psi \in \mathfrak{h}_{\omega}$ the functionals $a \mapsto\left\langle\pi_{\omega}(a) p_{0} \xi, \psi\right\rangle$ and $a \mapsto\left\langle\pi_{\omega}(a)(\mathbb{1}-\right.$ $\left.\left.p_{0}\right) \xi, \psi\right\rangle$ on $M$ are respectively the normal and singular parts of the functional $a \mapsto\left\langle\pi_{\omega}(a) \xi, \psi\right\rangle$. In particular, the triples $\left(p_{0} \pi_{\omega}\right.$, $\left.p_{0} \mathfrak{h}_{\omega}, p_{0} \Omega_{\omega}\right)$ and $\left(\left(\mathbb{1}-p_{0}\right) \pi_{\omega},\left(\mathbb{1}-p_{0}\right) \mathfrak{h}_{\omega},\left(\mathbb{1}-p_{0}\right) \Omega_{\omega}\right)$ are copies of the GNS representations of $\omega_{\mathrm{n}}$ and $\omega_{\mathrm{s}}$ respectively.

(ii) $\omega_{0}: a \mapsto\left\langle\pi_{\omega}(a)\left(\Omega_{\omega}-\Omega_{0}\right), \Omega_{\omega}-\Omega_{0}\right\rangle$ defines a positive functional on $M$ satisfying $\omega_{0}=\omega_{0} \circ \Phi$.

(b) Suppose that in addition $\operatorname{dim}(\mathcal{D})<\infty$.

(i) Then $\omega_{0}$ is a normal functional of the form $\omega_{0}=\tau\left(g^{1 / 2} \cdot g^{1 / 2}\right)$ for some $g \in \mathcal{D}_{+}$. Moreover $p_{0}\left(\Omega_{\omega}-\Omega_{0}\right)=\Omega_{\omega}-\Omega_{0}$, and $p_{0} \Omega_{0}$ is the orthogonal projection of $p_{0} \Omega_{\omega}$ onto $\overline{p_{0}\left(\pi_{\omega}\left(A_{0}\right) \Omega_{\omega}\right)}$.

(ii) If $\omega$ is singular, then $\pi_{\omega}(f) \Omega_{\omega} \in \overline{\pi_{\omega}\left(A_{0}\right) \Omega_{\omega}}$ for any $f \in \mathcal{D}$.

(c) Suppose that $\operatorname{dim}(\mathcal{D})<\infty$ and $\Omega_{\omega} \notin \overline{\pi_{\omega}\left(A_{0}\right) \Omega_{\omega}}$. If $\omega_{0}$ is faithful on $\mathcal{D}$, then there is a sequence $\left\{a_{n}\right\} \subset A$ such that $\pi_{\omega}\left(a_{n}\right)\left(\Omega_{\omega}-\Omega_{0}\right)$ $\rightarrow p_{0} \Omega_{\omega}$.

Proof. (a)(i) This is essentially the content of [31, III.2.14].

(a)(ii) Let $\left(\pi_{\omega}, \mathfrak{h}_{\omega}, \Omega_{\omega}\right)$ and $\Omega_{0}$ be as in the hypothesis, and define a positive functional $\omega_{0}$ on $M$ by

$$
\omega_{0}: a \mapsto\left\langle\pi_{\omega}(a)\left(\Omega_{\omega}-\Omega_{0}\right), \Omega_{\omega}-\Omega_{0}\right\rangle .
$$

Let $f \in A_{0}$ be given. By construction

$$
\pi_{\omega}(f) \Omega_{\omega} \perp\left(\Omega_{\omega}-\Omega_{0}\right) .
$$


Since $A_{0}$ is an ideal, $\pi_{\omega}(f a) \Omega_{\omega} \in \overline{\pi_{\omega}\left(A_{0}\right) \Omega_{\omega}}$ for each $a \in A_{0}$. Since $\Omega_{0}$ belongs to $\overline{\pi_{\omega}\left(A_{0}\right) \Omega_{\omega}}$, we may of course select a sequence $\left\{b_{n}\right\} \subset A_{0}$ for which $\pi_{\omega}\left(b_{n}\right) \Omega_{\omega}$ converges to $\Omega_{0}$. Hence $\pi_{\omega}\left(f b_{n}\right) \Omega_{\omega}$ converges to $\pi_{\omega}(f) \Omega_{0}$. Thus $\pi_{\omega}(f) \Omega_{0} \in \overline{\pi_{\omega}\left(A_{0}\right) \Omega_{\omega}}$, which forces

$$
\pi_{\omega}(f) \Omega_{0} \perp\left(\Omega_{\omega}-\Omega_{0}\right) .
$$

From the last two centered equations it is now clear that $A_{0} \subset \operatorname{Ker}\left(\omega_{0}\right)$. Thus $\omega_{0}=\omega_{0} \circ \Phi$ on $A$ by the remarks preceding Proposition 2.1. Hence $\omega_{0}=\omega_{0} \circ \Phi$ on $M$ by Corollary 2.3 .

(b)(i) Since $\mathcal{D}$ is finite-dimensional, we can find $g \in \mathcal{D}_{+}$so that

$$
\omega_{0}(a)=\tau(g a) \quad \text { for all } a \in \mathcal{D} .
$$

Since $\omega_{0} \circ \Phi=\omega_{0}$, we conclude that for any $a \in M$,

$$
\omega_{0}(a)=\omega_{0}(\Phi(a))=\tau(g \Phi(a))=\tau(\Phi(g a))=\tau(g a),
$$

thereby establishing the first part of the claim.

For the second part, note that since $\omega_{0}$ is clearly normal, by (a)(i) we have

$$
0=\left\langle\pi_{\omega}(a)\left(\mathbb{1}-p_{0}\right)\left(\Omega_{\omega}-\Omega_{0}\right), \Omega_{\omega}-\Omega_{0}\right\rangle \quad \text { for all } a \in M .
$$

For $a=\mathbb{1}$ this yields $0=\left\|\left(\mathbb{1}-p_{0}\right)\left(\Omega_{\omega}-\Omega_{0}\right)\right\|$, or equivalently

$$
p_{0}\left(\Omega_{\omega}-\Omega_{0}\right)=\Omega_{\omega}-\Omega_{0} .
$$

From this fact, we may now conclude that

$$
\left\langle p_{0} \pi_{\omega}(a) \Omega_{\omega}, p_{0}\left(\Omega_{\omega}-\Omega_{0}\right)\right\rangle=\left\langle\pi_{\omega}(a) \Omega_{\omega}, \Omega_{\omega}-\Omega_{0}\right\rangle=0 \quad \text { for all } a \in A_{0} .
$$

Thus $p_{0}\left(\Omega_{\omega}-\Omega_{0}\right) \perp \overline{p_{0} \pi_{\omega}\left(A_{0}\right) \Omega_{\omega}}$. Now select a sequence $\left\{b_{n}\right\} \subseteq A_{0}$ so that $\pi_{\omega}\left(b_{n}\right) \Omega_{\omega} \rightarrow \Omega_{0}$. By continuity, $p_{0} \Omega_{0}=\lim _{n} p_{0} \pi_{\omega}\left(b_{n}\right) \Omega_{\omega} \in \overline{p_{0} \pi_{\omega}\left(A_{0}\right) \Omega_{\omega}}$. From these considerations it is clear that $p_{0} \Omega_{0}$ is the orthogonal projection of $p_{0} \Omega_{\omega}$ onto $\overline{p_{0} \pi_{\omega}\left(A_{0}\right) \Omega_{\omega}}$.

(b)(ii) If $\omega$ is singular, then

$0=\omega_{\mathrm{n}}(a b)=\left\langle\pi_{\omega}(a b) p_{0} \Omega_{\omega}, \Omega_{\omega}\right\rangle=\left\langle p_{0} \pi_{\omega}(b) \Omega_{\omega}, \pi_{\omega}\left(a^{*}\right) \Omega_{\omega}\right\rangle \quad$ for all $a, b \in M$.

Since $\Omega_{\omega}$ is cyclic, this is sufficient to force $p_{0}=0$. But then $\Omega_{\omega}-\Omega_{0}=$ $p_{0}\left(\Omega_{\omega}-\Omega_{0}\right)=0$ by (b)(i). As before select $\left\{b_{n}\right\} \subset A_{0}$ so that $\pi_{\omega}\left(b_{n}\right) \Omega_{\omega} \rightarrow$ $\Omega_{0}=\Omega_{\omega}$. For any $f \in \mathcal{D}$ the ideal property of $A_{0}$ then ensures that $\pi_{\omega}(f) \Omega_{\omega}=\lim _{n} \pi_{\omega}\left(f b_{n}\right) \Omega_{\omega} \in \overline{\pi_{\omega}\left(A_{0}\right) \Omega_{\omega}}$.

(c) Suppose that $\omega_{\mathrm{n}}$, the normal part of $\omega$, is of the form $\omega_{\mathrm{n}}=\tau(h \cdot)$ for some $h \in L^{1}(M)_{+}$. As noted earlier, $\left(p_{0} \pi_{\omega}, p_{0} \mathfrak{h}_{\omega}, p_{0} \Omega_{\omega}\right)$ is a copy of the GNS representation generated by $\omega_{\mathrm{n}}$. If we now compute the GNS representation of $\omega_{\mathrm{n}}$ from first principles, it is clear that $p_{0} \mathfrak{h}_{\omega}$ corresponds to the weighted Hilbert space $L^{2}(M, h)$ obtained by equipping $M$ with the inner product

$$
\langle a, b\rangle_{h}=\tau\left(h^{1 / 2} b^{*} a h^{1 / 2}\right), \quad a, b \in M,
$$


and taking the completion. Note that $L^{2}(M, h)$ can be identified unitarily, and as $M$-modules, with the closure of $M h^{1 / 2}$ in $L^{2}(M)$. For any $a \in M$ considered as an element of $L^{2}(M, h)$ we will write $\Psi_{a}$ instead of $a$. The canonical $*$-homomorphism representing $M$ as an algebra of bounded operators on $L^{2}(M, h)$ is of course given by defining

$$
\pi_{\mathrm{n}}(b) \Psi_{a}=\Psi_{b a}, \quad a, b \in M,
$$

and then extending this action to all of $L^{2}(M, h)$. Since $\omega_{\mathrm{n}}$ is normal, $\pi_{\mathrm{n}}$ (corresponding to $\left.p_{0} \pi_{\omega}\right)$ is $\sigma$-weakly continuous and satisfies $\pi_{\mathrm{n}}(M)=\pi_{\mathrm{n}}(M)^{\prime \prime}$. Thus $\operatorname{Ker}\left(\pi_{\mathrm{n}}\right)$ is a $\sigma$-weakly closed two-sided ideal, and hence we can find a central projection $e \in M$ so that $(\mathbb{1}-e) M=\operatorname{Ker}\left(\pi_{\mathrm{n}}\right)$. Restrict $\pi_{\mathrm{n}}$ to a *-isomorphism from $e M$ onto $\pi_{\mathrm{n}}(M)$. Then for any $a, b, c \in M$ we have

$$
\left\langle\pi_{\mathrm{n}}(c) \Psi_{a}, \Psi_{b}\right\rangle_{h}=\tau\left(h^{1 / 2} b^{*}(e c e) a h^{1 / 2}\right) .
$$

Let $\Psi^{(0)}$ denote the orthogonal projection of $\Psi_{\mathbb{1}}$ onto the closure of $\left\{\Psi_{a}\right.$ : $\left.a \in A_{0}\right\}$. (Note that $\Psi_{\mathbb{1}}$ and $\Psi^{(0)}$ of course correspond to $p_{0} \Omega_{\omega}$ and $p_{0} \Omega_{0}$ in parts (a) and (b) of the proof.) Since $L^{2}(M, h)$ may be viewed as a subspace of $L^{2}(M)$, let $F \in L^{2}(M)$ be the element corresponding to $\Psi^{(0)}$. It is easy to see that $e F=F$. From (a) and (b) we now have

$$
\omega_{0}=\left\langle\pi_{\mathrm{n}}(\cdot)\left(\Psi_{\mathbb{1}}-\Psi^{(0)}\right), \Psi_{\mathbb{1}}-\Psi^{(0)}\right\rangle_{h}=\tau\left(\left(h^{1 / 2} e-F^{*}\right) \cdot\left(h^{1 / 2} e-F\right)\right) .
$$

This in turn ensures that

$$
\left|h^{1 / 2} e-F^{*}\right|^{2}=g
$$

where $g$ is as in (b). Thus $h^{1 / 2} e-F \in M$. Since by assumption $\omega_{0}$ is faithful on $\mathcal{D}$, it follows that $\operatorname{Supp}(g)=\mathbb{1}$. Since $\mathcal{D}$ is finite-dimensional, $g$ must be invertible. But then $h^{1 / 2} e-F$ must also be invertible, by the last centered equation. (Recall that if $a b$ is invertible in a finite von Neumann algebra then both $a$ and $b$ are invertible.) The polar decomposition of $h^{1 / 2} e-F^{*}$ is of the form $h^{1 / 2} e-F^{*}=u g^{1 / 2}$ for some unitary $u \in M$. From this it is clear that

$$
\left(h^{1 / 2} e-F\right)^{-1}=u g^{-1 / 2} .
$$

Clearly $h^{1 / 2} u g^{-1 / 2} \in L^{2}(M)$. Hence we may select $\left\{a_{n}\right\} \subset M$ converging in $L^{2}(M)$ to $h^{1 / 2} u g^{-1 / 2}=h^{1 / 2}\left(h^{1 / 2} e-F\right)^{-1}$. By the previously established correspondences we then have

$$
\begin{aligned}
\left\|\Psi_{\mathbb{1}}-\pi_{\mathrm{n}}\left(a_{n}\right)\left(\Psi_{\mathbb{1}}-\Psi^{(0)}\right)\right\|_{h} & =\tau\left(\left|h^{1 / 2} e-\left(a_{n} e\right)\left(h^{1 / 2} e-F\right)\right|^{2}\right)^{1 / 2} \\
& \rightarrow \tau\left(\left|h^{1 / 2} e-h^{1 / 2} e\right|^{2}\right)^{1 / 2}=0 .
\end{aligned}
$$

This implies, in the notation of (a) and (b), that $\pi_{\omega}\left(a_{n}\right)\left(\Omega_{\omega}-\Omega_{0}\right) \rightarrow p_{0} \Omega_{\omega}$.

It remains to show that we may select $\left\{a_{n}\right\} \subset A$, or equivalently, that $h^{1 / 2} u g^{-1 / 2} \in[A]_{2}$. For this, it suffices by the $L^{2}$ density of $A+A^{*}$ to show 
that $h^{1 / 2} u g^{-1 / 2} \perp\left[A_{0}^{*}\right]_{2}$. So let $a \in A_{0}$ be given, and observe that

$$
\begin{aligned}
\tau\left(a h^{1 / 2} u g^{-1 / 2}\right) & =\tau\left(g^{-1} a h^{1 / 2} u g^{-1 / 2} g\right)=\tau\left(g^{-1} a h^{1 / 2} u g^{1 / 2}\right) \\
& =\tau\left(\left(g^{-1} a h^{1 / 2}\right)\left(h^{1 / 2} e-F^{*}\right)\right)=\tau\left(\left(h^{1 / 2} e-F^{*}\right)\left(g^{-1} a h^{1 / 2}\right)\right) \\
& =\left\langle\Psi_{g^{-1} a}, \Psi_{\mathbb{1}}-\Psi^{(0)}\right\rangle_{h}=0 .
\end{aligned}
$$

(The last equality follows from the ideal property of $A_{0}$ and the fact that $\Psi_{\mathbb{1}}-\Psi^{(0)}$ is orthogonal to $\left\{\Psi_{a}: a \in A_{0}\right\}$.) The claim therefore follows.

Corollary 3.3. Let $A$ be a maximal subdiagonal algebra with $\operatorname{dim}(\mathcal{D})$ $<\infty$. The following are equivalent:

(i) A has the F \& $M$ Riesz property.

(ii) Whenever $\omega$ annihilates $A_{0}$, the normal and singular parts, $\omega_{\mathrm{n}}$ and $\omega_{\mathrm{s}}$, separately annihilate $A_{0}$.

(iii) Whenever $\omega$ annihilates $A, \omega_{\mathrm{n}}$ and $\omega_{\mathrm{s}}$ separately annihilate $A_{0}$.

(iv) Whenever $\omega$ annihilates $A, \omega_{\mathrm{n}}$ and $\omega_{\mathrm{s}}$ separately annihilate $A$.

Proof. The implications (i) $\Rightarrow($ ii) $\Rightarrow$ (iii) are clear. If (iii) holds, let $\omega$ be a bounded linear functional which annihilates $A_{0}$. Since $\Phi$ is a normal map onto $\mathcal{D}$, and $\mathcal{D}$ is finite-dimensional, the functional defined by

$$
\omega_{\mathcal{D}}=\left.\omega\right|_{\mathcal{D}} \circ \Phi
$$

is normal. Then $\varrho=\omega-\omega_{\mathcal{D}}$ defines a functional which annihilates $A$. From (iii) we then find that $\varrho_{\mathrm{n}}$ and $\varrho_{\mathrm{s}}$ separately annihilate $A_{0}$. The normality of $\omega_{\mathcal{D}}$ ensures that

$$
\varrho_{\mathrm{n}}=\omega_{\mathrm{n}}-\omega_{\mathcal{D}}, \quad \varrho_{\mathrm{s}}=\omega_{\mathrm{s}}
$$

Since by construction $\omega_{\mathcal{D}}$ annihilates $A_{0}$, we conclude that $\omega_{\mathrm{n}}$ and $\omega_{\mathrm{s}}$ separately annihilate $A_{0}$. This proves (ii).

To prove the validity of (i), it remains to show that any singular functional $\omega$ which annihilates $A_{0}$ also annihilates $\mathcal{D}$. For such $\omega$, the "modulus" $|\omega|$ is still singular (see e.g. $[16,11]$, or the argument in the first part of the proof of the next theorem). Let $\left(\pi_{\omega}, \mathfrak{h}_{\omega}, \Omega_{\omega}\right)$ be the GNS representation of $|\omega|$. For each $a \in M$ we have $|\omega(a)|^{2} \leq\|\omega\||\omega|\left(a^{*} a\right)$. By a standard argument this implies that there exists a vector $\eta \in \mathfrak{h}_{\omega}$ such that

$$
\omega(\cdot)=\left\langle\pi_{\omega}(\cdot) \Omega_{\omega}, \eta\right\rangle .
$$

Let $d \in \mathcal{D}$ be given. By Lemma 3.2(b)(ii) we may select a sequence $\left\{f_{n}\right\} \subset$ $A_{0}$ so that $\pi_{\omega}(d) \Omega_{\omega}=\lim _{n} \pi_{\omega}\left(f_{n}\right) \Omega_{\omega}$. But then

$$
\omega(d)=\left\langle\pi_{\omega}(d) \Omega_{\omega}, \eta\right\rangle=\lim _{n}\left\langle\pi_{\omega}\left(f_{n}\right) \Omega_{\omega}, \eta\right\rangle=\lim _{n} \omega\left(f_{n}\right)=0
$$

as required.

The equivalence with (iv) is now obvious.

TheOREM 3.4. Let $A$ be a maximal subdiagonal algebra. Then $A$ has the $F \& M$ Riesz property if and only if $\operatorname{dim}(\mathcal{D})<\infty$. 
Proof. We proved one direction in Proposition 3.1. For the other, let $\omega$ be a bounded linear functional on $M$ which annihilates $A_{0}$, and let $\omega_{\mathrm{n}}$ and $\omega_{\mathrm{s}}$ be the normal and singular parts of $\omega$. Write $\omega_{\mathrm{n}}=\tau(h \cdot)$ for some $h \in L^{1}(M)$. We extend $\omega, \omega_{\mathrm{n}}$, and $\omega_{\mathrm{s}}$ uniquely to normal functionals on the enveloping von Neumann algebra (the double commutant in the universal representation) and define $|\omega|,\left|\omega_{n}\right|$, and $\left|\omega_{\mathrm{s}}\right|$ to be the absolute values of these extensions restricted to $M$. Then from for example [16] (cf. [11, Proposition 7]) applied to $\omega$ and $\tau$, we deduce that as functionals on $M$, $\left|\omega_{\mathrm{n}}\right|$ and $\left|\omega_{\mathrm{s}}\right|$ are respectively the normal and singular parts of $|\omega|$, and that $|\omega|=\left|\omega_{\mathrm{n}}\right|+\left|\omega_{\mathrm{s}}\right|$. We note from [9, p. 270] that there is no danger of confusion as regards the absolute values of $\omega_{\mathrm{n}}$ since the absolute value of $\omega_{\mathrm{n}}$ as a functional on $M$ and as a functional on the enveloping von Neumann algebra coincide on $M$. Now consider the positive functional $\varrho$ given by

$$
\varrho=\tau+|\omega| .
$$

Let $\left(\pi_{\varrho}, \mathfrak{h}_{\varrho}, \Omega_{\varrho}\right)$ be the GNS representation constructed from $\varrho$, and define $\varrho_{0}$ by $\varrho_{0}(a)=\left\langle\pi_{\varrho}(a)\left(\Omega_{\varrho}-\Omega_{0}\right), \Omega_{\varrho}-\Omega_{0}\right\rangle$, where $\Omega_{0}$ is the orthogonal projection of $\Omega_{\varrho}$ onto the closure of $\left\{\pi_{\varrho}(a) \Omega_{\varrho}: a \in A_{0}\right\}$. For any $f \in A_{0}$ and any $d \in \mathcal{D}_{+}$, by construction we have

$$
\begin{aligned}
\left\|\pi_{\varrho}\left(d^{1 / 2}\right)\left(\Omega_{\varrho}-\pi_{\varrho}(f) \Omega_{\varrho}\right)\right\|^{2} & =\varrho\left(\left|d^{1 / 2}(\mathbb{1}-f)\right|^{2}\right) \geq \tau\left(\left|d^{1 / 2}(\mathbb{1}-f)\right|^{2}\right) \\
& =\tau\left(d-d f-f^{*} d+\left|d^{1 / 2} f\right|^{2}\right) \\
& =\tau\left(d+\left|d^{1 / 2} f\right|^{2}\right) \geq \tau(d) .
\end{aligned}
$$

On selecting a sequence $\left\{f_{n}\right\} \subset A_{0}$ so that $\pi_{\varrho}\left(f_{n}\right) \Omega_{\varrho} \rightarrow \Omega_{0}$, it follows that $\varrho_{0}(d)=\left\|\pi_{\varrho}\left(d^{1 / 2}\right)\left(\Omega_{\varrho}-\Omega_{0}\right)\right\|^{2} \geq \tau(d)$. Hence $\varrho_{0}$ is faithful on $\mathcal{D}$, and $\Omega_{\varrho} \neq \Omega_{0}$. Thus we may apply all of Lemma 3.2 to $\left(\pi_{\varrho}, \mathfrak{h}_{\varrho}, \Omega_{\varrho}\right)$.

Next notice that for each $a$ in the enveloping von Neumann algebra we have

$$
|\omega(a)|^{2} \leq\|\omega\||\omega|\left(a^{*} a\right) \leq\|\omega\| \varrho\left(a^{*} a\right) .
$$

Thus on restricting to elements of $M$, and employing a standard argument, this implies that there exists a vector $\eta \in \mathfrak{h}_{\varrho}$ such that

$$
\omega(\cdot)=\left\langle\pi_{\varrho}(\cdot) \Omega_{\varrho}, \eta\right\rangle .
$$

Now consider the related functional

$$
\widetilde{\omega}(\cdot)=\left\langle\pi_{\varrho}(\cdot)\left(\Omega_{\varrho}-\Omega_{0}\right), \eta\right\rangle .
$$

Select a sequence $\left\{f_{n}\right\} \subset A_{0}$ so that $\pi_{\varrho}\left(f_{n}\right) \Omega_{\varrho} \rightarrow \Omega_{0}$. Let $a \in A_{0}$ be given. Since $A_{0}$ is an ideal, and since $\omega$ annihilates $A_{0}$, we conclude that

$$
\begin{aligned}
\widetilde{\omega}(a) & =\left\langle\pi_{\varrho}(a)\left(\Omega_{\varrho}-\Omega_{0}\right), \eta\right\rangle=\lim _{n}\left\langle\pi_{\varrho}\left(a\left(\mathbb{1}-f_{n}\right)\right) \Omega_{\varrho}, \eta\right\rangle \\
& =\lim _{n} \omega\left(a\left(\mathbb{1}-f_{n}\right)\right)=0 .
\end{aligned}
$$

Thus $\widetilde{\omega}$ also annihilates $A_{0}$. 
By Lemma 3.2(c) we can find a sequence $\left\{a_{n}\right\} \subset A$ with $\pi_{\varrho}\left(a_{n}\right)\left(\Omega_{\varrho}-\Omega_{0}\right)$ $\rightarrow p_{0} \Omega_{\varrho}$. Let $a \in A_{0}$ be given. Since $A_{0}$ is an ideal, and since $\widetilde{\omega}$ annihilates $A_{0}$, we may now conclude that

$$
\omega_{\mathrm{n}}(a)=\left\langle\pi_{\varrho}(a) p_{0} \Omega_{\varrho}, \eta\right\rangle=\lim _{n}\left\langle\pi_{\varrho}\left(a a_{n}\right)\left(\Omega_{\varrho}-\Omega_{0}\right), \eta\right\rangle=\lim _{n} \widetilde{\omega}\left(a a_{n}\right)=0 .
$$

Thus $\omega_{\mathrm{n}}$ annihilates $A_{0}$. But then so does $\omega_{\mathrm{s}}=\omega-\omega_{\mathrm{n}}$. It now follows from Corollary 3.3 that $A$ has the F \& M Riesz property.

Corollary 3.5. If $A$ is a maximal subdiagonal algebra with $\mathcal{D}$ finitedimensional, and if $\omega \in M^{*}$ annihilates $A+A^{*}$, then $\omega$ is singular.

Proof. Since $A$ has the F \& M Riesz property, $\omega_{\mathrm{n}}$ annihilates $A$. Similarly, since $A^{*}$ has the $\mathrm{F} \& \mathrm{M}$ Riesz property, $\omega_{\mathrm{n}}$ annihilates $A^{*}$. Since $A$ is subdiagonal, $\omega_{\mathrm{n}}=0$.

Corollary 3.6. If $A$ has the $F \& B$ Riesz property, then any positive functional on $M$ which annihilates $A_{0}$ is normal.

Proof. If $\omega$ is a state on $M$ which annihilates $A_{0}$, and if $A$ has the $\mathrm{F} \&$ M Riesz property, then the (positive) singular part of $\omega$ is 0 since it must annihilate $\mathbb{1}$.

4. The Gleason-Whitney theorem. We now look at some properties which are stronger than the normal state extension property. We say that an extension in $M^{\star}$ of a functional in $A^{\star}$ is a Hahn-Banach extension if it has the same norm. If $A$ is a weak* closed subalgebra of $M$ then we say that $A$ has property (GW1) if every Hahn-Banach extension to $M$ of any normal functional on $A$ is normal on $M$. We say that $A$ has property (GW2) if there is at most one normal Hahn-Banach extension to $M$ of any normal functional on $A$. We say that $A$ has the Gleason-Whitney property $(\mathrm{GW})$ if it has (GW1) and (GW2). This is simply saying that there is a unique Hahn-Banach extension to $M$ of any normal functional on $A$, and this extension is normal. Of course normal functionals on $A$ or on $M$ have to be of the form $\tau(g \cdot)$ for some $g \in L^{1}(M)$.

Theorem 4.1. If $A$ is a tracial subalgebra of $M$ then $A$ is maximal subdiagonal if and only if it has property (GW2). If $\mathcal{D}$ is finite-dimensional, then $A$ is maximal subdiagonal if and only if it has property $(\mathrm{GW})$.

Proof. Suppose that $A$ has property (GW2). To show that $A$ is maximal subdiagonal, it suffices to show that if $g \in L^{1}(M)$ with $\tau\left(g\left(A+A^{*}\right)\right)=0$, then $g=0$. By considering real and imaginary parts we may assume that $g=g^{*}$. Then $\tau(|g| \cdot)$ and $\tau((|g|+g) \cdot)$ are positive normal functionals on $M$ which agree on $A$. They are also Hahn-Banach extensions, since the norm of a positive functional is achieved at 1 . Thus by (GW2), these functionals agree on $M$, and so $|g|+g=|g|$. That is, $g=0$. 
In the remainder of the proof suppose that $A$ is maximal subdiagonal. Suppose that $f, g \in L^{1}(M)$ correspond to two normal Hahn-Banach extensions to $M$ of a given functional on $A$. Then $\|f\|_{1}=\|g\|_{1}$, and this quantity equals the norm of the restriction to $A$. We have $\tau((f-g) A)=0$; since $A$ is subdiagonal it follows from $\left[28\right.$, Lemma 4] that $h=g-f \in\left[A_{0}\right]_{1}$. In order to establish (GW2), we need to show that $h=0$. Since $\operatorname{Ball}(A)$ is weak* compact, and since $\|f\|_{1}$ equals the norm of the above-mentioned restriction to $A$, there exists $a \in A$ of norm 1 with $\tau(f a)=\|f\|_{1}$. It is evident that

$$
|a f|^{2}=f^{*} a^{*} a f \leq f^{*} f=|f|^{2} .
$$

Now $0 \leq T \leq S$ in $L^{p}(M)$ implies that $T^{1 / 2} \leq S^{1 / 2}$ (see e.g. [29, Lemma 2.3]; we thank David Sherman for this reference). It follows that $|a f| \leq|f|$. On the other hand, $\tau(|f|)=\tau(f a)=\tau(a f) \leq \tau(|a f|)$. Thus $\||f|-|a f|\|_{1}=$ $\tau(|f|-|a f|)=0$, and so $|f|=|a f|$. The functional $\psi=\tau(a f \cdot)$ on $M$ must be positive since $\psi(\mathbb{1})=\tau(a f)=\tau(|f|)=\tau(|a f|)=\|\psi\|$. Thus af $\geq 0$, and $a f=|a f|=|f|$.

Since $h \in\left[A_{0}\right]_{1}$ we have

$$
\tau((f+h) a)=\tau(f a)=\|f\|_{1}=\|g\|_{1}=\|f+h\|_{1} .
$$

An argument similar to that of the last paragraph shows that $a(f+h)=$ $|f+h| \geq 0$. Thus $a h$ is self-adjoint. Since $h \in\left[A_{0}\right]_{1}$ it is easy to see that $\tau(a h A)=0$. Therefore from the self-adjointness of $a h$ one may deduce that $\tau\left(a h\left(A+A^{*}\right)\right)=0$. Because $A$ is subdiagonal, it follows that $a h=0$. Thus

$$
|f|=a f=a(f+h)=|f+h| .
$$

Let $e$ be the left support projection of $a$. Then $e^{\perp}$ is the projection onto $\operatorname{Ker}\left(a^{*}\right)$. We have $|f| e^{\perp}=f^{*} a^{*} e^{\perp}=0$. It follows that $f e^{\perp}=0$. Thus

$$
0=e^{\perp} f^{*} f e^{\perp}=e^{\perp}|f+h|^{2} e^{\perp}=e^{\perp}(f+h)^{*}(f+h) e^{\perp}=e^{\perp} h^{*} h e^{\perp} .
$$

Hence $h e^{\perp}=0$. To show that $h e=0$, we reproduce the ideas in the argument in the second paragraph of the proof. Namely, note that $\left|(f a)^{*}\right|^{2} \leq\left|f^{*}\right|^{2}$, so that $\left|(f a)^{*}\right| \leq\left|f^{*}\right|$. But $\tau\left(\left|f^{*}\right|\right)=\|f\|_{1}=\tau(f a) \leq \tau\left(\left|(f a)^{*}\right|\right)$, and as before this shows that $\left|(f a)^{*}\right|=\left|f^{*}\right|$. Then also $\tau(f a)=\tau\left(\left|(f a)^{*}\right|\right)$, and as before this shows that $f a \geq 0$. Similarly, $(f+h) a \geq 0$. So $h a$ is again self-adjoint, and this implies as before that $h a=0$. Thus $h e=0$, and so $h=h e+h e^{\perp}=0$ as required.

Now suppose that, in addition, $\mathcal{D}$ is finite-dimensional, and that $\varrho$ is a Hahn-Banach extension of a normal functional $\omega$ on $A$. By basic functional analysis, $\omega$ is the restriction of a normal functional $\widetilde{\omega}$ on $M$. We may write $\varrho=\varrho_{\mathrm{n}}+\varrho_{\mathrm{s}}$, where $\varrho_{\mathrm{n}}$ and $\varrho_{\mathrm{s}}$ are respectively the normal and singular parts, and $\|\varrho\|=\left\|\varrho_{\mathrm{n}}\right\|+\left\|\varrho_{\mathrm{s}}\right\|$. Then $\varrho-\widetilde{\omega}$ annihilates $A$, and hence by Corollary 3.3 and our F. and M. Riesz theorem both the normal and singular parts, $\varrho_{\mathrm{n}}-\widetilde{\omega}$ and $\varrho_{\mathrm{s}}$ respectively, annihilate $A$. In particular $\varrho_{\mathrm{n}}=\omega$ on $A$. But this implies 
that

$$
\left\|\varrho_{\mathrm{n}}\right\|+\left\|\varrho_{\mathrm{s}}\right\|=\|\varrho\|=\|\omega\| \leq\left\|\varrho_{\mathrm{n}}\right\| .
$$

We conclude that $\varrho_{\mathrm{s}}=0$. Thus $A$ also satisfies (GW1), and hence (GW).

As an immediate consequence, one sees that a tracial algebra $A$ is maximal subdiagonal iff every normal contractive unital $B(H)$-valued map has at most one normal positive $B(H)$-valued extension to $M$.

There is another (simpler) variant of the Gleason-Whitney theorem $[17$, p. 305], which transfers more easily to our setting:

THEOREM 4.2. Let $A$ be a maximal subdiagonal subalgebra of $M$ with $\mathcal{D}$ finite-dimensional. If $\omega$ is a normal functional on $M$ then $\omega$ is the unique Hahn-Banach extension of its restriction to $A+A^{*}$. In particular, $\|\omega\|=$ $\left\|\omega_{\mid A+A^{*}}\right\|$ for any $\omega \in M_{*}$.

Proof. Let $\varrho$ be a Hahn-Banach extension of the restriction of $\omega$ to $A+A^{*}$. We may write $\varrho=\varrho_{\mathrm{n}}+\varrho_{\mathrm{s}}$, where $\varrho_{\mathrm{n}}$ and $\varrho_{\mathrm{s}}$ are respectively the normal and singular parts, and $\|\varrho\|=\left\|\varrho_{\mathrm{n}}\right\|+\left\|\varrho_{\mathrm{s}}\right\|$. Then $\varrho-\omega$ annihilates $A+A^{*}$. By Corollary 3.5, $\varrho_{\mathrm{n}}-\omega=(\varrho-\omega)_{n}=0$. As in the last part of the previous proof, this implies that $\varrho_{\mathrm{s}}=0$. So $\varrho=\varrho_{\mathrm{n}}=\omega$.

REMARK. If $g \in L^{1}(M)$, and $\omega=\tau(g \cdot)$, then the last result shows that $\|g\|_{1}$ is the norm of the restriction of $\omega$ to $A+A^{*}$.

Corollary 4.3 (Kaplansky density theorem for subdiagonal algebras). Let $A$ be a maximal subdiagonal subalgebra of $M$ with $\mathcal{D}$ finite-dimensional. Then the unit ball of $A+A^{*}$ is weak* dense in $\operatorname{Ball}(M)$.

Proof. If $C$ is the unit ball of $A+A^{*}$, it follows from the last remark that the pre-polar of $C$ is $\operatorname{Ball}\left(M_{\star}\right)$. By the bipolar theorem, $C$ is weak* dense in $\operatorname{Ball}(M)$.

REMARK. We do not know if the last few results hold without the assumption that $\mathcal{D}$ be finite-dimensional. We also remark that there are $B(H)$ valued versions of these results.

5. Szegö and Kolmogorov theorems for $L^{p}(M)$. Arveson formulated the Szegő theorem for $L^{2}(M)$ in terms of the Kadison-Fuglede determinant $\Delta(\cdot)$. The long-standing open question of whether general maximal subdiagonal algebras satisfy the Szegő theorem for $L^{2}(M)$ was eventually settled in the affirmative in [21]. We will now extend this result to $L^{p}(M)$. We refer the reader to $[1,4]$ for the properties of the Kadison-Fuglede determinant which we shall need.

LEMmA 5.1. $\Delta\left(b^{p}\right)=\Delta(b)^{p}$ for $p \geq 1$ and $b \in M_{+}$.

Proof. By the multiplicativity of $\Delta$, the relation clearly holds for dyadic rationals. We may assume that $0 \leq b \leq 1$. In this case, by the functional 
calculus it is clear that $b^{q} \leq b^{p}$ if $0<p \leq q$. If $q$ is any dyadic rational larger than $p$ then

$$
\Delta(b)^{q}=\Delta\left(b^{q}\right) \leq \Delta\left(b^{p}\right) .
$$

It follows that $\Delta(b)^{p} \leq \Delta\left(b^{p}\right)$. Replacing $p$ by $1 / p$, we have $\Delta\left(b^{p}\right)^{1 / p} \leq$ $\Delta\left(\left(b^{p}\right)^{1 / p}\right)=\Delta(b)$, which gives the other direction.

Theorem 5.2 (An $L^{p}$ variant of Szegö's theorem). Suppose that $A$ is maximal subdiagonal, and $1 \leq p<\infty$. If $h \in L^{1}(M)_{+}$then $\Delta(h)=$ $\inf \left\{\tau\left(h|a+d|^{p}\right): a \in A_{0}, d \in \mathcal{D}, \Delta(d) \geq 1\right\}$.

Proof. We set

$$
\begin{aligned}
\mathcal{S}_{p} & =\left\{|a|^{p}: a \in A, \Delta(\Phi(a)) \geq 1\right\}, \\
\mathcal{S} & =\left\{a^{*} a: a \in A^{-1}, \Delta(a) \geq 1\right\} .
\end{aligned}
$$

By the modification in [4, Proposition 3.5] of a trick of Arveson's from [1, Theorem 4.4.3], it suffices to show that the closure of $\mathcal{S}_{p}$ equals the closure of $\mathcal{S}$. First we show that $\mathcal{S} \subset \mathcal{S}_{p}$. Indeed, if $b \in \mathcal{S}$ then $b$ is invertible, and therefore so is $b^{1 / p}$. Since $A$ has factorization, there is an $a \in A^{-1}$ with $|a|=b^{1 / p}$. By Lemma 5.1 and Jensen's formula $[1,21]$ we have

$$
\Delta(\Phi(a))=\Delta(a)=\Delta(|a|)=\Delta\left(b^{1 / p}\right)=\Delta(b)^{1 / p} \geq 1 .
$$

Hence $b=|a|^{p} \in \mathcal{S}_{p}$.

Suppose that $b \in \mathcal{S}_{p}$. If $b=|a|^{p}$ where $\Delta(\Phi(a)) \geq 1$ then by Jensen's inequality $[1,21]$ we have $\Delta(a)=\Delta(|a|) \geq 1$. Hence by Lemma 5.1 we have $\Delta(b) \geq 1$. If $n \in \mathbb{N}$ then since $A$ has factorization, there exists a $c \in A^{-1}$ with $b+(1 / n) 1=c^{*} c$. Thus

$$
\Delta(c)^{2}=\Delta\left(b+\frac{1}{n} 1\right) \geq \Delta(b) \geq 1 .
$$

Hence $b+(1 / n) 1=c^{*} c \in \mathcal{S}$, and we deduce that $b \in \overline{\mathcal{S}}$. Hence $\overline{\mathcal{S}}_{p} \subset \overline{\mathcal{S}}$.

Note that the following generalized Kolmogorov theorem is not true for all maximal subdiagonal algebras. For example, take $A=M=L^{\infty}[0,1]$.

THEOREM 5.3. Suppose that $A$ is an antisymmetric maximal subdiagonal algebra. If $h \in L^{1}(M)_{+}$then $\inf \left\{\tau\left(h|\mathbb{1}+f|^{2}\right): f \in A_{0}+A_{0}^{*}\right\}$ is either $\tau\left(h^{-1}\right)^{-1 / 2}$ if $h^{-1}$ exists in the sense of unbounded operators and is in $L^{1}(M)$; or 0 if $h^{-1} \notin L^{1}(M)$. More generally, if $1 \leq p<\infty$ then $\inf \left\{\tau\left(\left|(\mathbb{1}+f) h^{1 / p}\right|^{p}\right): f \in A_{0}+A_{0}^{*}\right\}$ is either 0 if $h^{-1} \notin \overline{L^{1 /(p-1)}}(M)$, or $\tau\left(h^{-1 /(p-1)}\right)^{1 / p-1}$ if $h^{-1} \in L^{1 /(p-1)}(M)$.

Proof. We formally follow the proof of Forelli as adapted in [30, p. 247]. Let $h \in L^{1}(M)_{+}$, and $1 / p+1 / q=1$. Define $L^{p}(M, h)$ to be the completion in $L^{p}(M)$ of $M h^{1 / p}$. Note that if $e$ is the support projection of a positive $x \in L^{p}(M)$ then it is well known (see e.g. [20, Lemma 2.2]) that $L^{p}(M) e$ 
equals the closure of $M x$ in $L^{p}(M)$. Hence $L^{p}(M, h)=L^{p}(M) e$, where $e$ is the support projection of $h$. Now for any projection $e \in M$ it is an easy exercise to prove that the dual of $L^{p}(M) e$ is $e L^{q}(M)$ (see e.g. [20]). It follows that the dual of $L^{p}(M, h)$ is $L^{q}(M, h)$.

If $k \in L^{p}(M, h)$ then $k h^{1 / q} \in L^{p}(M) L^{q}(M) \subset L^{1}(M)$. We view $A_{0}+A_{0}^{*}$ in $L^{p}(M, h)$ as its image $\left(A_{0}+A_{0}^{*}\right) h^{1 / p}$, and let $N$ be the annihilator of this in $L^{q}(M, h)$. That is, $g \in N$ iff $g \in L^{q}(M, h)$ and

$$
0=\tau\left(h^{1 / p}\left(A_{0}+A_{0}^{*}\right) g\right)=\tau\left(\left(A_{0}+A_{0}^{*}\right) g h^{1 / p}\right) .
$$

Since $g h^{1 / p} \in L^{1}(M)$ the last equation holds iff $g h^{1 / p}=c \mathbb{1}$, where $c$ is a constant. Since $h$ is self-adjoint, if $c \neq 0$ then it follows that $h^{-1 / p}$ exists in the sense of unbounded operators, and its closure is the constant multiple $d g \in L^{q}(M)$, where $d=c^{-1}$. (Since we are in the finite case, there is no difficulty with $\tau$-measurability here, which is automatic [32].) If $c=0$ then $g h^{1 / p}=0$, which implies that $g=0$. To see the last statement note that if $h^{1 / p}$ is viewed as a self-adjoint unbounded operator on a Hilbert space $H$, and if $e$ is its support projection, which equals the support projection of $h^{1 / q}$, then $e h^{1 / p}=h^{1 / p}$, and so $h^{1 / p} e=h^{1 / p}$. Since $g \in \overline{M h^{1 / q}}$, we have $g e=g$. However, $g e=0$ since $g h^{1 / p}=0$. Thus if $g$ has norm 1 then $c \neq 0$, $h^{-1 / p} \in L^{q}(M)$ and $|d|=\left\|h^{-1 / p}\right\|_{L^{q}(M)}=\tau\left(h^{-q / p}\right)^{1 / q}$.

The infimum in the theorem is the $p$ th power of the norm of $\mathbb{1}$ in the quotient space of $L^{p}(M, h)$ modulo the closure of $A_{0}+A_{0}^{*}$. Since the dual of this quotient is $\left(A_{0}+A_{0}^{*}\right)^{\perp}=N$, this infimum equals the $p$ th power of $\sup \left\{\left|\tau\left(g h^{1 / p}\right)\right|: g \in N,\|g\|_{L^{q}(M)} \leq 1\right\}$. This equals 0 if no $g \in N$ has norm 1; otherwise it equals $\tau\left(h^{-q / p}\right)^{-1 / q}=\tau\left(h^{-1 /(p-1)}\right)^{-1 / q}$ by the above. Indeed, the infimum is 0 iff $\tau\left(g h^{1 / p}\right)=0$ for all $g \in N$. Since $g h^{1 / p}$ is constant, this occurs iff $g h^{1 / p}=0$, which as we saw above happens iff $g=0$. Thus the infimum is 0 iff $N=(0)$ iff $\left(A_{0}+A_{0}^{*}\right) h^{1 / p}$ is dense in $L^{p}(M, h)$. Since $h^{1 / p} \in L^{p}(M, h)$, the latter condition implies that there is a sequence $\left(g_{n}\right)$ in $A_{0}+A_{0}^{*}$ with $g_{n} h^{1 / p} \rightarrow h^{1 / p}$ in $p$-norm. If $h^{-1 / p} \in L^{q}(M)$ then by Hölder's inequality we have $\tau\left(\left|g_{n}-\mathbb{1}\right|\right) \rightarrow 0$, which is impossible since $1=\left|\tau\left(g_{n}-\mathbb{1}\right)\right| \leq \tau\left(\left|g_{n}-\mathbb{1}\right|\right)$.

Acknowledgements. We thank Mike Marsalli for many valuable discussions, and Marius Junge for a helpful insight concerning Theorem 2.7.

Note added in proof. Other variants of Theorem 5.2 have been established in the forthcoming paper [7]. These variants seem more useful when generalizing many of the classical results concerning outer functions to the noncommutative $H^{p}$ context. For all practical purposes, the results of that paper therefore complete the noncommutative extension of the basic "generalized" Hardy space theory for abstract function algebras surveyed in $[30]$. 


\section{References}

[1] W. B. Arveson, Analyticity in operator algebras, Amer. J. Math. 89 (1967), 578-642.

[2] - On groups of automorphisms of operator algebras, J. Funct. Anal. 15 (1974), $217-243$.

[3] D. P. Blecher and L. E. Labuschagne, Logmodularity and isometries of operator algebras, Trans. Amer. Math. Soc. 355 (2003), 1621-1646.

[4] - - - Characterizations of noncommutative $H^{\infty}$, Integral Equations Operator Theory, to appear.

[5] -, - A Beurling theorem for noncommutative $L^{p}$, J. Operator Theory, to appear.

[6] - - - Von Neumann algebraic $H^{p}$ theory, to appear.

[7] -, 一, Applications of the Fuglede-Kadison determinant: Szegö's theorem and outers for noncommutative $H^{p}$, arXiv:math.OA/0609662.

[8] D. P. Blecher and C. Le Merdy, Operator Algebras and their Modules-an Operator Space Approach, Oxford Univ. Press, 2004.

[9] J. Dixmier, $C^{*}$-Algebras, North-Holland, Amsterdam, 1977.

[10] R. Exel, Maximal subdiagonal algebras, Amer. J. Math. 110 (1988), 775-782.

[11] -, The F. and M. Riesz theorem for $C^{*}$-algebras, J. Operator Theory 23 (1990), 351-368.

[12] T. Fack and H. Kosaki, Generalized s-numbers of $\tau$-measurable operators, Pacific J. Math. 123 (1986), 269-300.

[13] T. W. Gamelin, Uniform Algebras, 2nd ed., Chelsea, New York, 1984.

[14] A. M. Gleason and H. Whitney, The extension of linear functionals defined on $H^{\infty}$, Pacific J. Math. 12 (1962), 163-182.

[15] H. Helson and D. Lowdenslager, Prediction theory and Fourier series in several variables, Acta Math. 99 (1958), 165-202.

[16] M. Henle, A Lebesgue decomposition theorem for $C^{*}$-algebras, Canad. Math. Bull. 15 (1972), 87-91.

[17] K. Hoffman, Analytic functions and logmodular Banach algebras, Acta Math. 108 (1962), 271-317.

[18] —, Banach Spaces of Analytic Functions, Dover, 1988.

[19] K. Hoffman and H. Rossi, Function theory and multiplicative linear functionals, Trans. Amer. Math. Soc. 116 (1965), 536-543.

[20] M. Junge and D. Sherman, Noncommutative $L^{p}$-modules, J. Operator Theory 53 (2005), 3-34.

[21] L. E. Labuschagne, A noncommutative Szegö theorem for subdiagonal subalgebras of von Neumann algebras, Proc. Amer. Math. Soc. 133 (2005), 3643-3646.

[22] G. Lumer, Analytic functions and Dirichlet problems, Bull. Amer. Math. Soc. 70 (1964), 98-104.

[23] M. Marsalli and G. West, Noncommutative $H^{p}$-spaces, J. Operator Theory 40 (1998), 339-355.

[24] M. McAsey, P. Muhly, and K.-S. Saito, Nonselfadjoint crossed products (invariant subspaces and maximality), Trans. Amer. Math. Soc. 248 (1979), 381-409.

[25] T. Nakazi and Y. Watatani, Invariant subspace theorems for subdiagonal algebras, J. Operator Theory 37 (1997), 379-395.

[26] G. Pisier and Q. Xu, Non-commutative $L^{p}$-spaces, in: Handbook of the Geometry of Banach Spaces, Vol. 2, W. B. Johnson and J. Lindenstrauss (eds.), North-Holland, Amsterdam, 2003, 1459-1517.

[27] G. Popescu, Entropy and multivariable interpolation, Mem. Amer. Math. Soc. 184 (2006), no. 868. 
[28] K.-S. Saito, A note on invariant subspaces for finite maximal subdiagonal algebras, Proc. Amer. Math. Soc. 77 (1979), 348-352.

[29] L. M. Schmitt, The Radon-Nikodým theorem for $L^{p}$-spaces of $W^{*}$-algebras, Publ. Res. Inst. Math. Sci. 22 (1986), 1025-1034.

[30] T. P. Srinivasan and J.-K. Wang, Weak*-Dirichlet algebras, in: Function Algebras, F. T. Birtel (ed.), Scott and Foresman, 1966, 216-249.

[31] M. Takesaki, Theory of Operator Algebras, Vol. 1, Springer, New York, 1979.

[32] M. Terp, $L^{p}$ spaces associated with von Neumann algebras, notes, Math. Institute, Copenhagen Univ., 1981.

[33] L. Zsidó, Spectral and ergodic properties of the analytic generators, J. Approx. Theory 20 (1977), 77-138.

Department of Mathematics

University of Houston

Houston, TX 77204-3008, U.S.A.

E-mail: dblecher@math.uh.edu
Department of Mathematical Sciences

P.O. Box 392

0003 Unisa, South Africa

E-mail: labusle@unisa.ac.za

Received March 17, 2006

Revised version October 30, 2006 